\title{
Collective pulsational velocity broadening due to gravity modes as a physical explanation for macroturbulence in hot massive stars
}

\author{
C. Aerts ${ }^{1,2}$, J. Puls ${ }^{3}$, M. Godart ${ }^{4}$, and M.-A. Dupret ${ }^{4}$
}

\author{
1 Instituut voor Sterrenkunde, Katholieke Universiteit Leuven, Celestijnenlaan 200D, 3001 Leuven, Belgium \\ e-mail: conny@ster.kuleuven.be \\ 2 IMAPP, Department of Astrophysics, Radboud University Nijmegen, PO Box 9010, 6500 GL Nijmegen, the Netherlands \\ 3 Universitäts-Sternwarte, Scheinerstrasse 1, 81679 München, Germany \\ 4 Institut d'Astrophysique et Géophysique, Université de Liège, allée du Six Août 17, 4000 Liège, Belgium
}

Received 26 June 2008 / Accepted 17 September 2009

\section{ABSTRACT}

\begin{abstract}
Aims. We aimed at finding a physical explanation for the occurrence of macroturbulence in the atmospheres of hot massive stars, a phenomenon found in observations for more than a decade but that remains unexplained.

Methods. We computed time series of line profiles for evolved massive stars broadened by rotation and by hundreds of low-amplitude nonradial gravity-mode pulsations which are predicted to be excited for evolved massive stars.

Results. In general, line profiles based on macrotubulent broadening can mimic those subject to pulsational broadening. In several cases, though, good fits require macroturbulent velocities that pass the speed of sound for realistic pulsation amplitudes. Moreover, we find that the rotation velocity can be seriously underestimated by using a simple parameter description for macroturbulence rather than an appropriate pulsational model description to fit the line profiles.

Conclusions. We conclude that macroturbulence is a likely signature of the collective effect of pulsations. We provide line diagnostics and their typical values to decide whether or not pulsational broadening is present in observed line profiles, as well as a procedure to avoid an inaccurate estimation of the rotation velocity.
\end{abstract}

Key words. line: profiles - techniques: spectroscopic - stars: atmospheres - supergiants - stars: early-type - stars: variables: general

\section{Introduction of the phenomenon of macroturbulence}

Stars are gaseous bodies that transfer hydrogen into helium through nuclear burning in their core during most of their lives. A variety of evolved stars results after the exhaustion of the core hydrogen burning. It is the birth mass of the star that determines which evolutionary path the evolved star will follow. Here, we are concerned with stars whose birth mass is above ten solar masses. Such massive stars undergo subsequent nuclear burning cycles until their core is composed of iron, after which they collapse as supernovae. While this broad picture of stellar evolution is well understood and in agreement with various types of observations, we still lack knowledge of important aspects of the physics and dynamics inside massive stars and of their consequences for the stellar life.

One particular shortcoming in the description of the physics of stellar atmospheres of massive stars is the need to introduce an ad-hoc velocity field, termed macroturbulence, at the stellar surface in order to bring the observed shape of spectral lines into agreement with observations. While evidence for the occurrence of such macroturbulence in hot stars has been established for more than a decade (Howarth et al. 1997), there is still no physical explanation for this phenomenon. This unsatisfactory situation has become ever more problematic as the data improved in quality in terms of resolving power and signal-to-noise $(S / N)$ ratio and in quantity in terms of the number of stars that have been studied with high-resolution spectroscopy. It turns out that the macroturbulent velocities required to explain high-quality observations are supersonic in many of the studied stars, which would point to highly dynamical atmospheric motion whose cause is unknown (Ryans et al. 2002; Lefever et al. 2007; Markova \& Puls 2008). Here, we provide a natural physical explanation for this phenomenon in terms of the collective effect of numerous stellar pulsations of low amplitude.

Velocity fields of very different scales occur in the atmospheres of stars. Apart from the rotational velocity which can vary from zero speed up to the critical value, line synthesis codes also include a certain amount of microturbulence (of order a few $\mathrm{km} \mathrm{s}^{-1}$ ) to bring the observed profiles in the spectra of stars into agreement with the data. Microturbulence is defined as a phenomenon related to velocity fields with scales shorter than the mean free path of the photons in the atmosphere (e.g., Gray 2005 for a thorough explanation). Microturbulence and rotation are usually treated as time-independent processes leading to line profile broadening.

In contrast to microturbulence, macroturbulence refers to velocity fields with a scale larger than the mean free path of the photons (with mesoturbulence as the intermediate situation e.g., Gray 1978). Macroturbulence was mainly introduced and studied in the context of cool stars (e.g, Gray 1973, 1975, 1978). Various descriptions have been proposed in the literature (see Gray 2005 for an overview), among which an isotropic model and a radial-tangential model are the most common ones. Both these models will be considered here.

Values for the micro- and macroturbulence are usually derived from line-profile fits of single snapshot spectra. Here we focus on such applications to massive hot stars, whose 
microturbulent velocities are usually below $15 \mathrm{~km} \mathrm{~s}^{-1}$ (e.g., McErlean et al. 1998; Villamariz \& Herrero 2000). The published values of macroturbulence, on the other hand, are usually well above this value, reaching up to $90 \mathrm{~km} \mathrm{~s}^{-1}$ (Lefever et al. 2007; Markova \& Puls 2008). An important omission so far in the derivation of macroturbulence is that time-dependent velocity phenomena also occur, besides rigid surface rotation and turbulence. The best known example of such a phenomenon is stellar pulsation, which causes asymmetric line-profile variations (e.g., Aerts \& De Cat 2003). A natural step is thus to investigate whether the needed macroturbulence may be connected with the omission of pulsational broadening in the line synthesis codes used for fundamental parameter estimation. In fact, for pulsating stars along the main sequence, one also needs to add some level of macroturbulence whenever one ignores (some of) the detected pulsations in line-profile fitting of time-resolved or averaged spectra (e.g., Morel et al. 2006). We investigate this hypothesis in the present paper.

\section{Computations of pulsationally broadened spectral line profiles}

Massive stars are exposed to pulsations during several phases of their life. On or near the main sequence, these pulsations are usually driven by a heat mechanism acting in the metal opacity bump at a temperature near 200000 degrees (e.g., Cox et al. 1992; Pamyatnyh 1999). In the recent and rapidly growing research field of asteroseismology, observed pulsations are exploited by scientists to probe the poorly known physical processes inside stars (e.g., Cunha et al. 2007; Aerts et al. 2009), as was done in helioseismology for the Sun (e.g., Gough et al. 1996). Asteroseismology was proven to be a valid tool to study the interior of massive main-sequence stars (e.g., Aerts et al. 2003) and may be a unique opportunity to probe the internal layers, including the deep convection zone around the hydrogenburning shell, of evolved stars as well. The discovery of gravitymode pulsations in the B1Ib supergiant HD 163899 from spacebased high-precision photometry measured with the Canadian space mission MOST (Saio et al. 2006) and in a sample of $40 \mathrm{~B}$ supergiants (Lefever et al. 2007) are steps in this direction. We refer the reader to Aerts et al. (2009) for a thorough description of stellar pulsation in all of its aspects, including the particular properties of the eigenfrequencies and eigenfunctions of pressure and gravity modes.

It is well known that stellar pulsations imply a timedependent variation of the shape of spectral lines (e.g., Aerts \& De Cat 2003, for a review, Chaps. 4 and 6 of Aerts et al. 2009). Despite this, the estimation of the rotational and macroturbulent velocity in evolved massive stars has so far usually been done from a single snapshot of the stellar spectrum, and assuming that no time-dependent phenomena are present. Here, we investigate to what extent stellar pulsations affect the estimation of the surface rotation and macroturbulent velocities when ignoring pulsations, as was often done in the literature. For this, we computed numerous sets of line profiles due to pulsations expected in B supergiants.

\subsection{Input for the simulations}

Simulating line profile variations due to excited oscillations of a star requires the following steps:

1. the computation of an equilibrium stellar structure model;
2. the computation of the excited oscillation frequencies of the stellar model;

3 . the computation of the oscillation eigenfunctions in the lineforming region of the stellar atmosphere;

4. the computation of the observed line profile as seen by a distant observer, whose line of sight is inclined with respect to the symmetry axis of the oscillations.

For points 1 and 2 we considered a realistic case and computed a stellar evolution model that is representative of the evolved pulsating B1Ib star HD 163899 with the Code Liégeois d'Évolution Stellaire (Scuflaire et al. 2008). This model has the following parameters: $T_{\text {eff }}=18200 \mathrm{~K}, \log g=3.05 R / R_{\odot}=17.8$, $\log \left(L / L_{\odot}\right)=4.5 M / M_{\odot}=13, Z=0.02$ and an age of thirtheen million years. It approximates well the position of HD 163889 in the Hertzsprung-Russell diagram (Saio et al. 2006). We determined its excited pulsation modes of azimuthal order zero with a non-adiabatic pulsation code MAD (Dupret 2001). We considered all modes up to degree ten, as it is well-known that partial geometrical cancellation effects increase with increasing mode degree (e.g., Chap. 6 of Aerts et al. 2009, for a full description of these effects) and, moreover, we needed to keep the computation time feasible. We found 241 modes with degree $\ell$ from 1 to 10 to be excited. All of them are gravity modes, with frequencies ranging from 0.08 to 0.68 cycles per day, a result typical for gravity modes.

Regarding points 3 and 4, it was shown by De Ridder et al. (2002) that the temperature and gravity variations in the lineforming region due to the pulsations do not affect the line profile variations of a non-rotating star appreciably. This conclusion was based on the computation of temperature and gravity variations for the stellar interior and for the atmosphere, and applying a matching in a connecting layer which separates the region where the diffusion approximation breaks down from the stellar interior where it is valid (Dupret et al. 2002). This justifies the use of the basic line profile theory as described in Aerts et al. (1992). That framework allows the computation of the velocity eigenvectors of the modes in the line-of-sight for a linear limbdarkening law. It makes use of the velocity perturbations in a single line-forming layer of the atmosphere to predict the line profile variations, while ignoring temperature, gravity, and rotational effects. Ideally, rotational effects should be included in the computations, given that the ratio of the rotation to pulsation frequencies can be of order one. Theories including a non-adiabatic treatment of rotational effects due to the Coriolis force are available for the stellar interior, where the diffusion approximation is valid (e.g., Lee 2001; Townsend 2005). A study like the one by Dupret et al. (2002) which treats the velocity and temperature perturbations of a rotating star in the very outer atmosphere is not yet available. Developing it is beyond the scope of the present work, which is simply to generate profiles due to pulsations with properties similar to those observed and interpret them as macroturbulence.

Rotation splits the frequencies of modes into $2 \ell+1$ multiplet components (e.g., Aerts et al. 2009). As there is currently no theory to provide us with the excitation of rotationally split modes, nor with the amplitudes of the modes, we assume that all modes with azimuthal orders $m$ ranging from $-\ell$ to $\ell$ are excited with equal amplitudes in the line-forming region and we assume these amplitudes to be $v_{\mathrm{p}} \propto(\ell+1)^{-1}$ in the notation by Aerts et al. (1992). We checked that changing these assumptions does not alter the conclusions presented here, by considering also the case where only axisymmetric or sectoral modes would be excited and by choosing different amplitude laws. Provided that a 

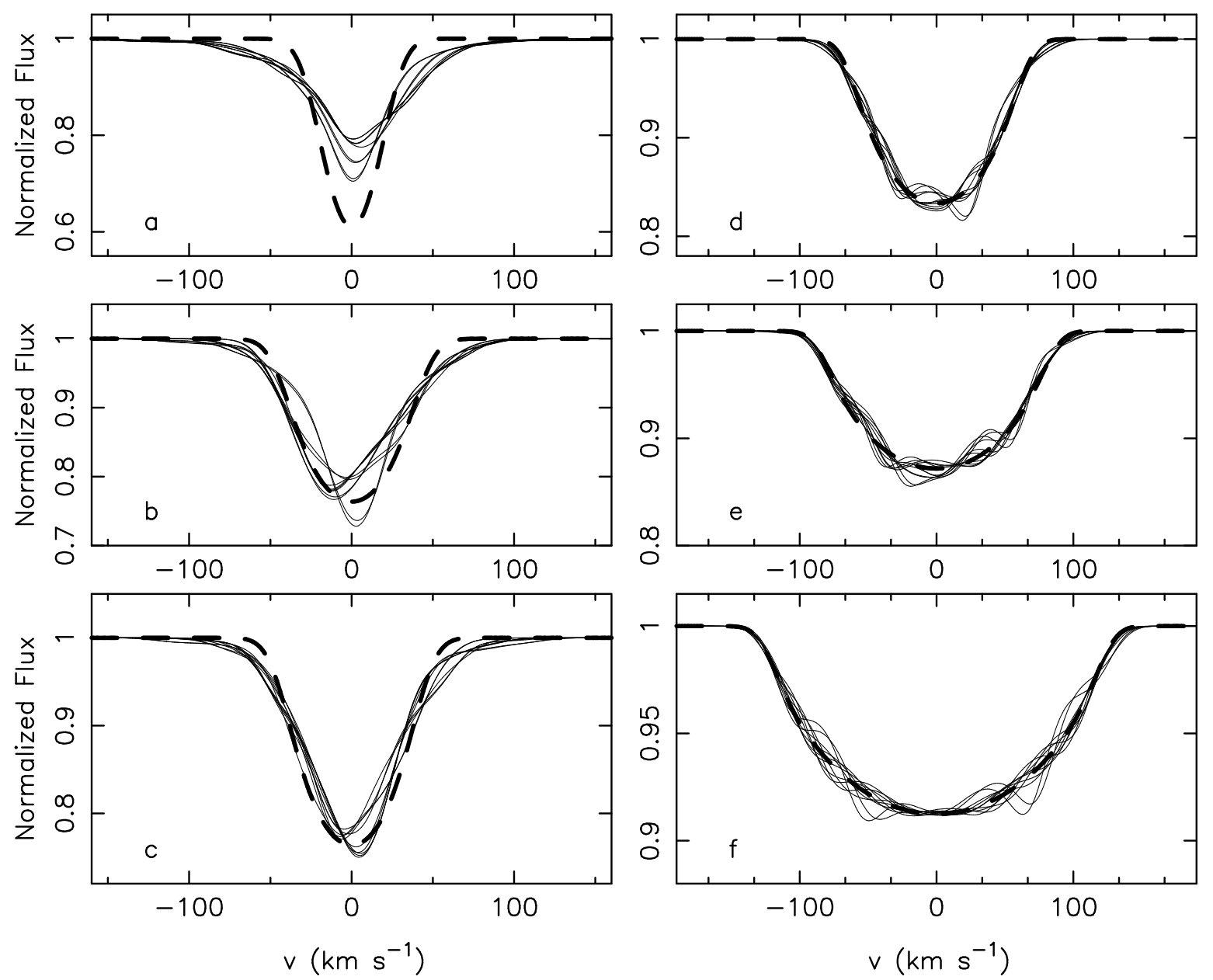

Fig. 1. Noiseless pulsationally and rotationally broadened profiles (thin lines) are compared with the profile without pulsational but with rotational broadening (dashed line). The input parameters are the stellar inclination angle, the amplitude of the individual modes, and the projected rotation velocity, $(i, a, v \sin i)$. Their values are as follows: a): $\left.\left(60^{\circ}, 1.0,25\right), \mathbf{b}\right):\left(60^{\circ}, 1.0,45\right)$, c): $\left.\left.\left.\left(20^{\circ}, 1.0,45\right), \mathbf{d}\right):\left(60^{\circ}, 0.5,65\right), \mathbf{e}\right):\left(60^{\circ}, 0.5,85\right), \mathbf{f}\right)$ : $\left(60^{\circ}, 0.2,125\right)$, where $a$ and $v \sin i$ are expressed in $\mathrm{km} \mathrm{s}^{-1}$ (see text for a definition of $a$ ). Note that the line features in panels d), e), f) become less visible as the $S / N$ ratio decreases. They essentially disappear for $S / N<100$ (see also Fig. 3).

sufficient number of modes are included in the line broadening computations (typically at least a few hundred), our conclusions remain the same and are thus independent of the adopted amplitude distribution. The conclusions are not dependent on the particular stellar model either.

In total, the 241 excited $m=0$ modes give rise to $2965 \mathrm{mul}$ tiplet components. We computed the collective effect of all these 2965 gravity modes on simulated line profiles. The simulations were made such as to mimic the effect on the Si III $4553 \AA$ line in the spectrum of a star with the fundamental parameters of HD 163899. In our simulations, we approximated the Si III $4553 \AA$ line by a Gaussian profile of width $10 \mathrm{~km} \mathrm{~s}^{-1}$ and equivalent width of $0.25 \AA$, and we adopted a linear limb darkening law with a fixed coefficient equal to 0.364 across the line. These values were also fixed when computing the fits to the pulsationally broadened profiles. In this way, we are sure that our conclusions on the macroturbulence are not affected by adopting a wrong microturbulence or by a varying limb darkening coefficient across the spectral line. We limited ourselves to tuning towards this one Si spectral line, since: (i) it is an important diagnostic line of intermediate strength; (ii) it is (almost) not contaminated by non-Gaussian broadening (such as Stark broadening in the case of hydrogen and helium lines) or wind effects; and (iii) it is the line selected for almost all of the pulsating early
B stars so far as it turned out to be best suited to derive their pulsation characteristics (Aerts \& De Cat 2003). On the other hand, our approximation of a constant Gaussian intrinsic line implies that our analysis is valid for any metal line of this width in the spectrum. We computed time series of profiles for 50 timings taken from a concrete line profile study (Štefl et al. 1999) with a total time span of 65 days.

We simulated various time series of 50 profiles each, taking into account pulsational and rotational broadening, besides the intrinsic broadening of the spectral line. In our computations, we considered five values of the projected rotation velocity $v \sin i$ $\left(25,45,65,85,125 \mathrm{~km} \mathrm{~s}^{-1}\right)$. We limited our study to this range of $v \sin i$, for which the equatorial rotation velocities remain below $50 \%$ of the critical velocity of the stellar model $\left(305 \mathrm{~km} \mathrm{~s}^{-1}\right)$. As can be seen in Fig. 1 of Aerts et al. (2004), this implies sufficiently small relative changes of the local radius, temperature, gravity, and luminosity to ignore the centrifugal force in the computation of the equilibrium structure model of the star.

We adopted an inclination angle $i$ between the rotational axis and the line-of-sight of $60^{\circ}$, but we also considered $20^{\circ}$ for the case of $v \sin i=45 \mathrm{~km} \mathrm{~s}^{-1}$. The symmetry axis of the pulsations was taken equal to the rotational axis, as is usually done in pulsation studies of non-magnetic stars. Regarding the pulsational broadening, we considered four distributions for the 


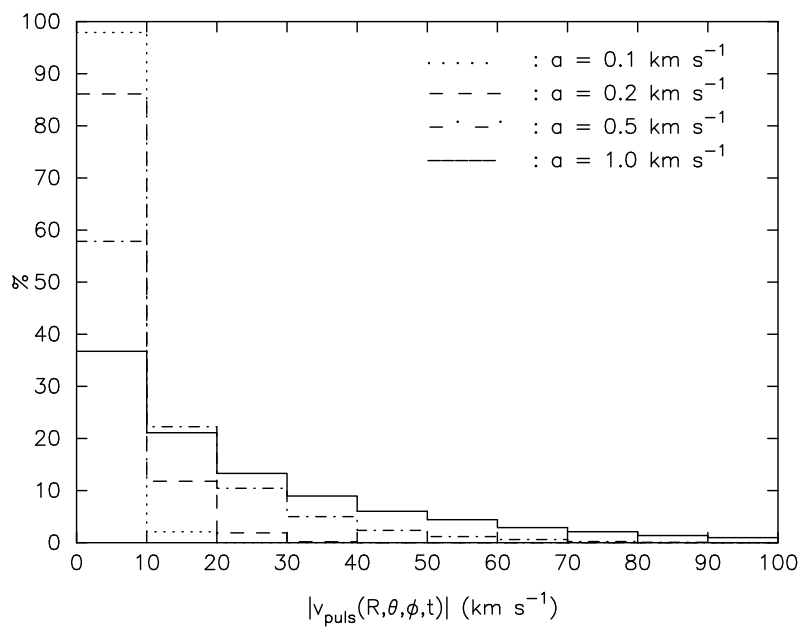

Fig. 2. Distribution of the projected pulsational velocity over the stellar surface as measured by a distant observer whose line-of-sight is inclined by $60^{\circ}$ with respect to the rotation axis of the star, for four distributions of the pulsational amplitudes $v_{\mathrm{p}}$ (see text for further explanation).

intrinsic amplitude of the modes in the line-forming region: $v_{\mathrm{p}}=a /(\ell+1)$ with $a=1.0,0.5,0.2,0.1 \mathrm{~km} \mathrm{~s}^{-1}$, again using the notation of Aerts et al. (1992). This means that the radial component of the pulsational velocity vector is proportional to $v_{\mathrm{p}}$ while the transversal component is proportional to $v_{\mathrm{p}} K$ with $K \equiv G M / \omega^{2} R^{3}$ with $G$ the gravitational constant, $M$ and $R$ the mass and radius of the star, and $\omega$ the angular frequency of the mode (see, e.g., Aerts et al. 2009). For the adopted model we consider here, the $K$-values of the considered modes range from 0.3 to 25 . The choice of these amplitude distributions was made to end up with a realistic peak-to-peak variation of the radial velocity as in published observed time series of the few supergiant B stars for which such data are available - see Figs. 5 and 6 in Kaufer et al. (1997), Figs. 2 and 3 in Prinja et al. (2004) and Fig. 2 in Markova et al. (2008). These studies have led to radialvelocity variations with peak-to-peak amplitudes between 5 and $20 \mathrm{~km} \mathrm{~s}^{-1}$. Our amplitude distributions for $v_{\mathrm{p}}$ were taken accordingly, i.e., the collective effect of the 2965 gravity modes with the amplitude distributions we adopted results in radial-velocity variations similar to the observed ranges (see $\langle v\rangle$ in Fig. 6 discussed in Sect. 2.2). In this way, we are sure to have generated realistic profile variations, irrespective of the limitations of the line profile theory discussed above. A summary of the input parameters of the simulated line profile sets, along with some of their computed quantities discussed below, is given in Table 1.

The radial velocity is an integrated quantity over the stellar surface. Pulsating stars have time-dependent asymmetric lineprofile variations. It is common to characterise the line profile shapes by their three lowest-order moments, which represent the centroid velocity $\langle v\rangle$, the width $\left\langle v^{2}\right\rangle$ and the skewness $\left\langle v^{3}\right\rangle$. A practical guide to compute these quantities, as well as their formal definition in terms of the surface velocity eigenfunctions, is provided in De Ridder et al. (2002) and more extensively in Chap. 6 of Aerts et al. (2009). Aerts et al. (1992) and Aerts (1996) provided a thorough discussion of these quantities and their suitability to interprete them in terms of pulsation theory, thus allowing an identification of the spherical wavenumbers $(\ell, m)$ from observed time series of moment variations. We computed these three quantities for the simulated line profiles, mainly to show their relation to the derived macroturbulent velocity values obtained when ignoring the pulsational broadening, as will be discussed in Sect. 2.2. The first moment $\langle v\rangle$ is the radial velocity of the star, integrated over the stellar disc, with respect to the centre of mass of the star (i.e. it varies around a value of zero during the pulsation cycle); it is thus directly comparable to the measured radial-velocity variations reported in the literature which are usually based on Gaussian fits to the profiles.

In order to end up with peak-to-peak radial-velocity variations of order $20 \mathrm{~km} \mathrm{~s}^{-1}$, as measured for several supergiant B stars from metal lines, numerous of the individual surface elements must experience a far larger individual pulsation velocity. In the case of radial pulsations and in the approximation of the adopted linear limb darkening law, this means that the entire surface moves up and down with a velocity of about $20 \times 1.5=30 \mathrm{~km} \mathrm{~s}^{-1}$. This also implies that measured radialvelocity variations above typically $40 \mathrm{~km} \mathrm{~s}^{-1}$ are the results of shock phenomena in the atmosphere of radial B-type pulsators, leading to a so-called "stillstand" in the radial-velocity curve in the case of radial modes. Examples can be found in Aerts et al. (1995), Saesen et al. (2005) and Briquet et al. (2009) for the $\beta$ Cep stars BW Vul, $\xi^{1}$ CMa and V1449 Aql, respectively. Such a stillstand was so far not observed for B supergiants, so we expect the majority of the surface elements to move subsonically (which does not imply that some elements may encounter supersonic speeds).

For non-radial gravity modes, a wide variety of surface velocities occurs across the stellar surface and shock phenomena are much harder to detect in integrated quantities, such as moments or equivalent widths. We show in Fig. 2 the distribution of the line-of-sight components of the total pulsational velocity vectors, which result from the addition of all the individual vectors of the 2965 modes, for each of the surface elements (denoted as $\left.\left|v_{\text {puls }}(R, \theta, \phi, t)\right|\right)$, for the four amplitude sets corresponding to the four $a$-values. The addition of the numerous eigenvectors can result in positive or negative projected velocity, depending on the phases of the modes and on the location $(R, \theta, \phi)$ on the surface. We expect that, in most of the surface points and for most of the timings, positive and negative contributions tend to lead to a limited value of the overall pulsation velocity due to cancelling of positive and negative mode velocities, since we assumed there to be no phase relation between the modes. It can be seen from Fig. 2 that, for all four amplitude sets, the majority of the surface elements indeed are seen to move subsonically. For $a=1.0 \mathrm{~km} \mathrm{~s}^{-1}$, supersonic speeds in the line-of-sight are encountered for a considerable fraction of the surface elements, but still in less than half of them such that the radial-velocity variations remain below $20 \mathrm{~km} \mathrm{~s}^{-1}$ (see Fig. 6 discussed in Sect. 2.2). The adopted amplitude distributions thus lead to realistic peakto-peak amplitudes for the radial velocity. In this way, we are sure not to overestimate the effects of pulsations on the derivation of the macroturbulent velocity values.

We considered profile sets without noise and with white noise resulting in $S / N$ ratios of 200 and 500. This brings the total number of simulates profiles to 3600 (50 timings, 6 combinations $(v \sin i, i), 4$ amplitude distributions and 3 noise levels). Examples are provided in Fig. 1 and lead to the conclusion that some of the simulated profiles are considerably affected by the collective effect of the gravity modes. In particular, the line wings are broader than those that would occur for a star that does not have pulsations.

\subsection{Estimation of the macroturbulent velocities}

Various possibilities to describe macroturbulence have been presented in the literature. We refer to Gray (2005) for a thorough 

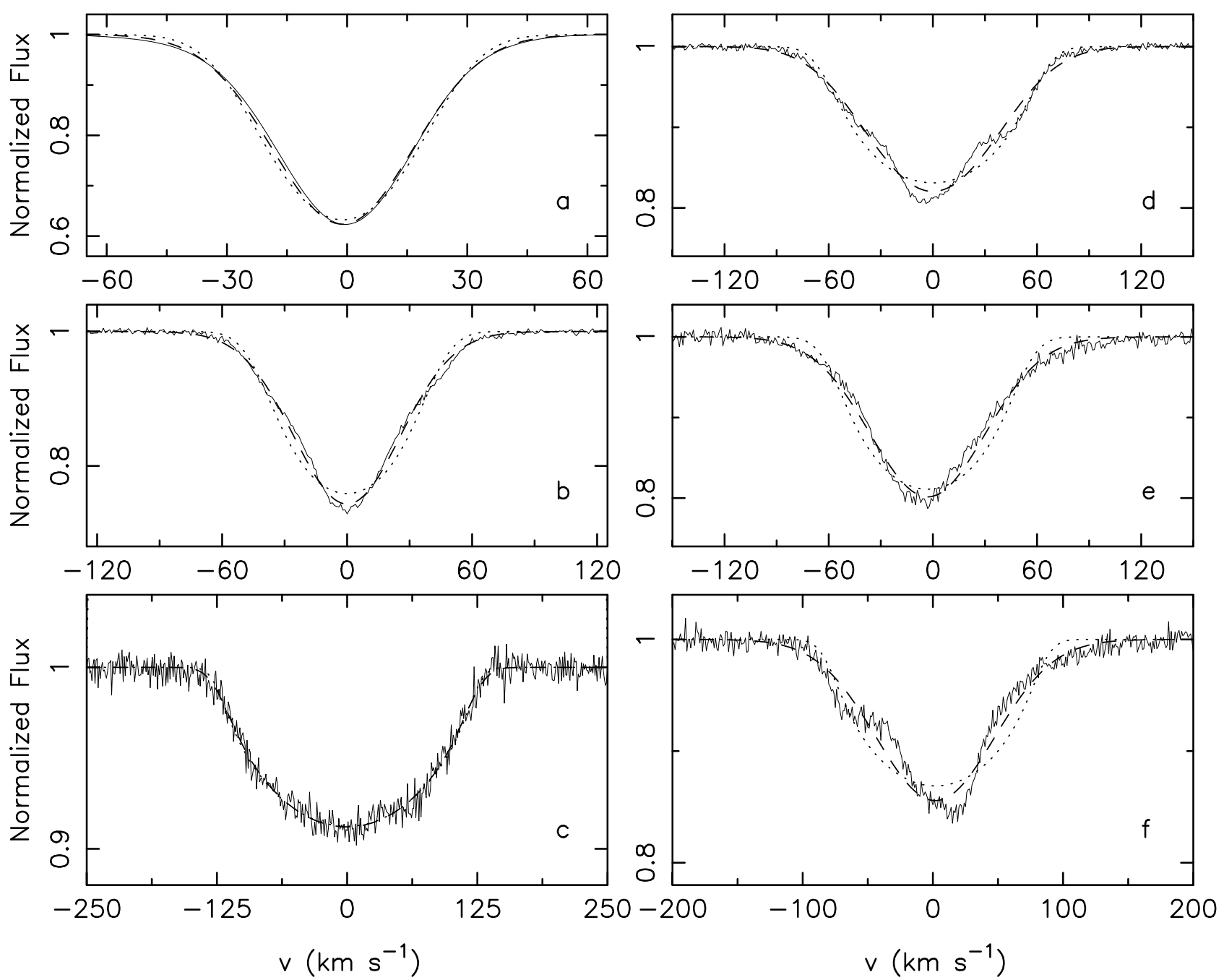

Fig. 3. Six pulsationally broadened profiles with different $S / N$ ratio (full lines) are compared with their best fit including both rotation and macroturbulence (dashed lines) and rotation alone (dotted line). The values for the input rotation velocity, the rotation velocity from a fit without macroturbulence, and from a fit with isotropic macroturbulence, $\left(v \sin i\right.$ (in), $v \sin i$ (fit, $\left.v_{\text {macro }}=0\right) ; v \sin i$ (fit), $v_{\text {macro }}$ ), are as follows: a): $(25,27 ; 8,14)$, b): $(45,44 ; 11,23)$, c): $(125,126 ; 125,10)$, d): $(65,64 ; 49,24), \mathbf{e}):(45,57 ; 5,32), \mathbf{f}):(85,82 ; 14,44)$, where all velocities are expressed in km ${ }^{-1}$. The fits without macroturbulence (dotted lines) lead to more reliable values of $v \sin i$ (see text for explanation).

discussion. In this work, we considered an isotropic macroturbulence described by a Gaussian velocity distribution (denoted as $A_{\text {ISO }}$ ), as well as an anisotropic description for which the radial and tangential velocity fields in general have a different amplitude denoted as $A_{\mathrm{R}}$ and $A_{\mathrm{T}}$ (a so-called radial-tangential modelsee Eq. (17.6), p. 433 in Gray 2005). For the anisotropic model fits, we considered the two extreme cases of allowing $A_{\mathrm{R}}$ to be free while $A_{\mathrm{T}}=0$ and $A_{\mathrm{R}}=0$ while $A_{\mathrm{T}}$ was allowed to take any value. In this way, each of the three models for the macroturbulence is described by one free parameter.

For all the simulated profiles, we determined $v \sin i$ and the macroturbulence $v_{\text {macro }}$, while ignoring the presence of pulsational broadening, as is done in the literature, by adopting a goodness-of-fit approach. The normalized profiles broadened by both rotation and gravity-mode pulsations are denoted by $\left(\lambda_{j}, p_{1}\left(\lambda_{j}\right)\right)$ and those broadened by rotation and macroturbulence by $\left(\lambda_{j}, p_{2}\left(\lambda_{j}\right)\right)$, with $j=1, \ldots, N$ an index labelling the velocity pixels within the profile. For the computation of $p_{2}$ we considered each of the three options $A_{\mathrm{ISO}}, A_{\mathrm{R}}$, and $A_{\mathrm{T}}$. Each of the profiles $p_{1}$ and $p_{2}$ were given the same equivalent width. We computed the line deviation parameter, $\Sigma$, based on the classical statistical technique of residuals:

$\Sigma\left(v \sin i, v_{\text {macro }}\right) \equiv \sqrt{\frac{1}{N-1} \sum_{j=1}^{N}\left[p_{1}\left(\lambda_{j}\right)-p_{2}\left(\lambda_{j}\right)\right]^{2}}$.

This quantity is the standard deviation of the residual profile $\left|p_{1}-p_{2}\right|$, averaged over all velocity pixels in the line profile, expressed in continuum units. It is thus a measure of the fit quality, directly interpretable in terms of the $S / N$ ratio of measurements. The optimal choice of the parameters $\left(v \sin i, v_{\text {macro }}\right)$ is then found by carefully screening the 2-dimensional parameter space in $v \sin i$ and $v_{\text {macro }}$ (in steps of $1 \mathrm{~km} \mathrm{~s}^{-1}$ for each of $v \sin i$ and $\left.v_{\text {macro }}\right)$ and by identifying $\Sigma_{\mathrm{m}} \equiv \min _{\left(v \sin i, v_{\text {macro }}\right)} \Sigma$, where $v_{\text {macro }}$ can be any of $A_{\mathrm{ISO}}, A_{\mathrm{R}}$ or $A_{\mathrm{T}}$. Moreover, we allowed two options to fit the pulsationally broadened profile $p_{1}$ : the one such that the wavelength position of the minimum of $p_{1}$ and $p_{2}$ coincide and the one such that their first moments $\langle v\rangle$ are in best agreement. Note that these two options are equivalent only in the case of symmetric profiles. For each generated profile, we 
Table 1. Ranges of the intervals for the macroturbulence and moments of the 72 sets of simulated line profiles with pulsational broadening ( 3 values of the $S / N$ for each of the combinations $(i, v \sin i, a)$, with $v_{\mathrm{p}}=a /(\ell+1)$. See text for further explanation.

\begin{tabular}{ccccccc}
\hline \hline$i$ & $v \sin i$ & $a$ & $\begin{array}{c}v_{\text {macro }} \\
\mathrm{km} \mathrm{s}^{-1}\end{array}$ & $\begin{array}{c}\langle v\rangle \\
\mathrm{km} \mathrm{s}^{-1}\end{array}$ & $\begin{array}{c}\left\langle v^{2}\right\rangle \\
\mathrm{km}^{2} \mathrm{~s}^{-2}\end{array}$ & $\begin{array}{c}\left\langle v^{3}\right\rangle \\
\mathrm{km}^{3} \mathrm{~s}^{-3}\end{array}$ \\
\hline 20 & 45 & 0.1 & {$[0,14]$} & {$[-0.5,0.7]$} & {$[588,611]$} & {$[-920,1645]$} \\
20 & 45 & 0.2 & {$[0,21]$} & {$[-1.0,1.4]$} & {$[618,674]$} & {$[-1826,3287]$} \\
20 & 45 & 0.5 & {$[7,44]$} & {$[-2.4,3.6]$} & {$[788,1091]$} & {$[-7956,12931]$} \\
20 & 45 & 1.0 & {$[21,67]$} & {$[-4.8,7.1]$} & {$[1360,2543]$} & {$[-32298,64385]$} \\
60 & 25 & 0.1 & {$[0,7]$} & {$[-0.3,0.4]$} & {$[248,272]$} & {$[-558,544]$} \\
60 & 25 & 0.2 & {$[0,12]$} & {$[-0.7,0.7]$} & {$[255,328]$} & {$[-804,1586]$} \\
60 & 25 & 0.5 & {$[11,29]$} & {$[-1.6,1.8]$} & {$[326,691]$} & {$[-3872,10999]$} \\
60 & 25 & 1.0 & {$[24,56]$} & {$[-3.3,3.6]$} & {$[573,1942]$} & {$[-16257,59011]$} \\
60 & 45 & 0.1 & {$[0,8]$} & {$[-0.5,0.6]$} & {$[576,602]$} & {$[-971,1081]$} \\
60 & 45 & 0.2 & {$[0,17]$} & {$[-0.9,1.2]$} & {$[591,656]$} & {$[-1987,2804]$} \\
60 & 45 & 0.5 & {$[11,33]$} & {$[-2.3,2.9]$} & {$[688,1068]$} & {$[-8721,9546]$} \\
60 & 45 & 1.0 & {$[26,67]$} & {$[-4.7,5.9]$} & {$[982,2599]$} & {$[-4130429635]$} \\
60 & 65 & 0.1 & {$[0,23]$} & {$[-0.3,0.7]$} & {$[1095,1143]$} & {$[-1725,3343]$} \\
60 & 65 & 0.2 & {$[0,28]$} & {$[-0.7,1.5]$} & {$[1106,1225]$} & {$[-3999,6820]$} \\
60 & 65 & 0.5 & {$[6,53]$} & {$[-1.7,3.6]$} & {$[1176,1672]$} & {$[-12753,19100]$} \\
60 & 65 & 1.0 & {$[25,70]$} & {$[-3.3,7.3]$} & {$[1466,3266]$} & {$[-42861,56467]$} \\
60 & 85 & 0.1 & {$[0,14]$} & {$[-0.5,0.8]$} & {$[1793,1848]$} & {$[-3349,6224]$} \\
60 & 85 & 0.2 & {$[0,33]$} & {$[-1.0,1.7]$} & {$[1794,1912]$} & {$[-7028,11406]$} \\
60 & 85 & 0.5 & {$[12,61]$} & {$[-2.4,4.1]$} & {$[1853,2314]$} & {$[-20976,27259]$} \\
60 & 85 & 1.0 & {$[27,81]$} & {$[-4.7,8.3]$} & {$[2173,3772]$} & {$[-61905,93998]$} \\
60 & 125 & 0.1 & {$[0,22]$} & {$[-0.6,0.7]$} & {$[3745,3865]$} & {$[-8440,11892]$} \\
60 & 125 & 0.2 & {$[0,55]$} & {$[-1.1,1.4]$} & {$[3707,3956]$} & {$[-16857,23578]$} \\
60 & 125 & 0.5 & {$[14,79]$} & {$[-2.7,3.4]$} & {$[3665,4422]$} & {$[-44830,64179]$} \\
60 & 125 & 1.0 & {$[10,97]$} & {$[-5.5,6.7]$} & {$[3810,6007]$} & {$[-124040,166820]$} \\
\hline
\end{tabular}

performed these six fitting exercises, keeping in each case the fit with the lowest $\Sigma_{\mathrm{m}}\left(v \sin i, v_{\text {macro }}\right)$ as the best one. We then compared the parameters $\left(v \sin i, v_{\text {macro }}\right)$ of the best fitting profile $p_{2}$ with the input $v \sin i$ of $p_{1}$ and analysed the values of $v_{\text {macro }}$. Six prototypical examples of best fits are shown in Fig. 3.

In Fig. 4 we show the outcome of the fit to the 3600 simulated profiles, for the three models we considered for the macroturbulence. For all three models, it was found that the inclusion of an ad-hoc macroturbulence parameter leads to better fits than those obtained when only allowing rotational broadening, which has to be the case given that there is one more degree of freedom. This is visible from Fig. 5 where we show the distribution of $\Sigma_{\mathrm{m}}$ deduced from fits with and without allowing a parameter for macroturbulence. Figure 5 contains all simulated profiles; these two global distributions are the same as those for the five separate values of $v \sin i$, which is as expected given that the pulsational broadening was simply added to the rotational broadening without any coupling between the two. One would typically improve the fit quality obtained from eye inspection by incorporating macroturbulence for $\Sigma_{\mathrm{m}}>0.008$; this corresponds to the dotted lines in panels b,d,e,f in Fig. 3 whose counterparts with macroturbulence represented by the dashed lines imply a noticable reduction in $\Sigma_{\mathrm{m}}$ (for comparison, the dotted lines in panels a and $\mathrm{c}$ have $\Sigma_{\mathrm{m}}=0.0058$ and 0.0056 , respectively, and would probably not give rise to the introduction of macroturbulence). It can be seen from the distribution of $\Sigma_{\mathrm{m}}$ in Fig. 5 that the fit quality is very good for the large majority of profiles when allowing for macroturbulent broadening. In $89 \%$ of the cases, the fit with macroturbulence has $\Sigma_{\mathrm{m}}<0.008$. If we do not include macroturbulence, $59 \%$ of the fits have $\Sigma_{\mathrm{m}}<0.008$.

Returning to Fig. 4, we deduce that the lowest $\Sigma_{\mathrm{m}}$ values were reached for $A_{\mathrm{ISO}}, A_{\mathrm{R}}$, and $A_{\mathrm{T}}$ in 1224,1343 , and 1033 of the cases, so these descriptions are basically equivalent in appropriateness to mimic pulsational broadening. It can be seen from Fig. 4 that the radial model $A_{\mathrm{R}}$ needs higher values to achieve a good fit compared with the isotropic and tangential model. This is logical, because the pulsation velocities of the modes are predominantly horizontal in nature. There are some differences between the fit quality in a global sense for three considered models, but the main conclusion is that the missing broadening caused by the pulsations can often only be compensated for by quite large values of the macroturbulence.

In Fig. 6 we show the value of the macroturbulence with the lowest $\Sigma_{\mathrm{m}}$, along with the first and third velocity moment, of the simulated profiles. As in Fig. 4, this plot illustrates that the value of the macroturbulence can be very large, compatible with what is found in the literature, if one ignores pulsational broadening, even though the centroid velocity variations $\langle v\rangle$ induced by the pulsations are modest. The reason for this is that the line width is a function of the square of the velocity, and the line skewness is represented by $\left\langle v^{3}\right\rangle$. Thus, one needs to compensate the line width and line wing shape by a large value for the macroturbulence whenever one wants to achieve a good profile fit.

The reported absence of line asymmetries in the literature must be compatible with our physical line-broadening model. Most spectroscopic studies in which symmetric profiles are mentioned rely on visual inspection of only one spectrum, while line profile variability (and thus asymmetry) is almost always found when multiple-epoch observations are taken. Visual inspection of the fits in the top and bottom of the right column in Fig. 3 reveals line asymmetry from one snapshot spectrum, while the other four profiles might give the impression of being symmetric. Typically, the profiles simulated with $a=1.0 \mathrm{~km} \mathrm{~s}^{-1}$ would be detectable by visual inspection of the profiles. However, when one computes diagnostic line quantities, it often becomes obvious that the lines deviate from symmetry even if seemingly symmetric by eye. The best diagnostic parameters to characterise line asymmetry in the case of pulsations are the line moments. While line bisectors and velocity spans are often used in the cool star and exoplanet communities, such parameters are not 


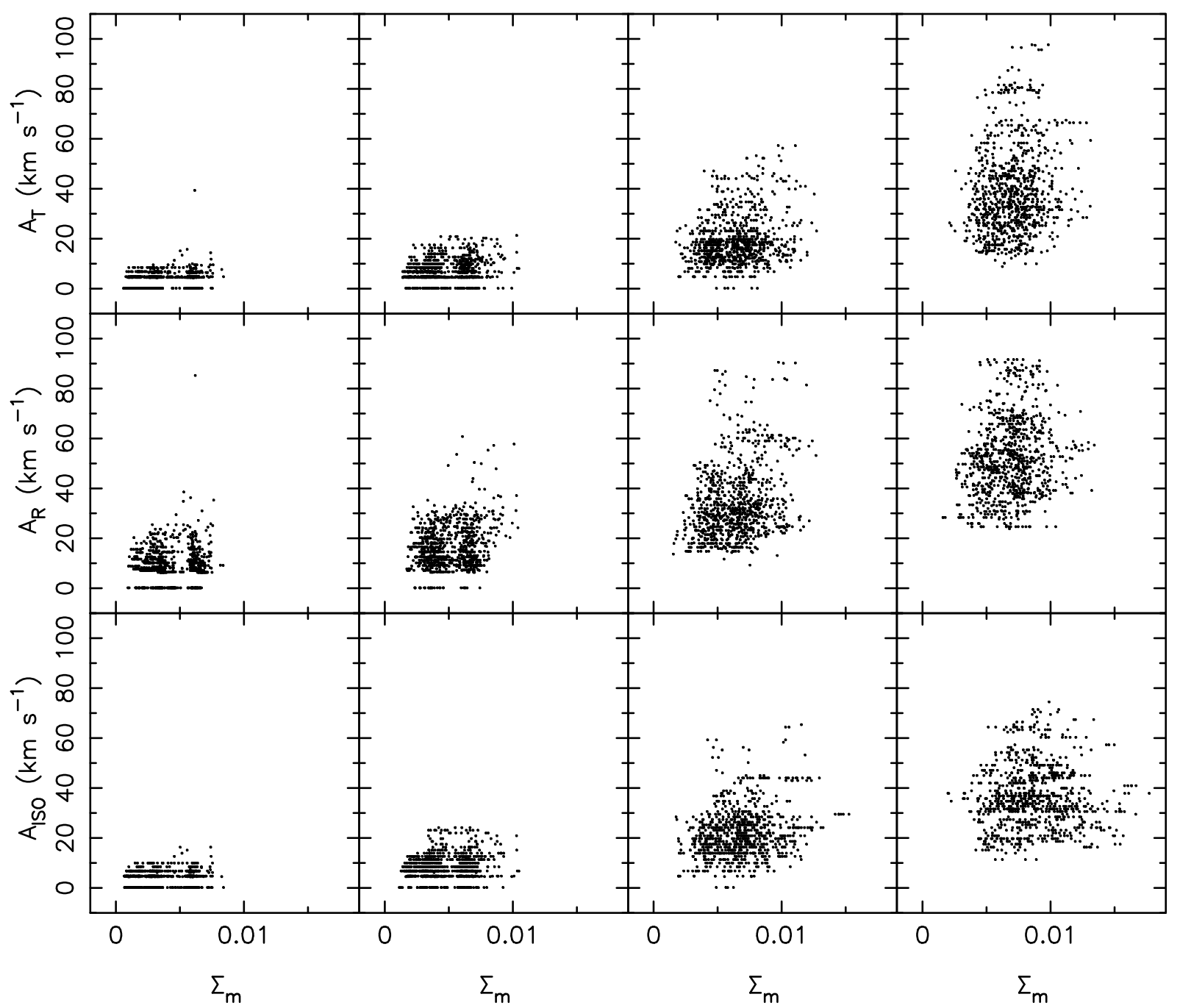

Fig. 4. $\Sigma_{\mathrm{m}}$ (in continuum units) for the three models of macroturbulence $A_{\mathrm{ISO}}, A_{\mathrm{R}}$, and $A_{\mathrm{T}}$, for the four amplitude distributions (left to right: $a=0.1,0.2,0.5,1.0 \mathrm{~km} \mathrm{~s}^{-1}$, see also Table 1$)$.

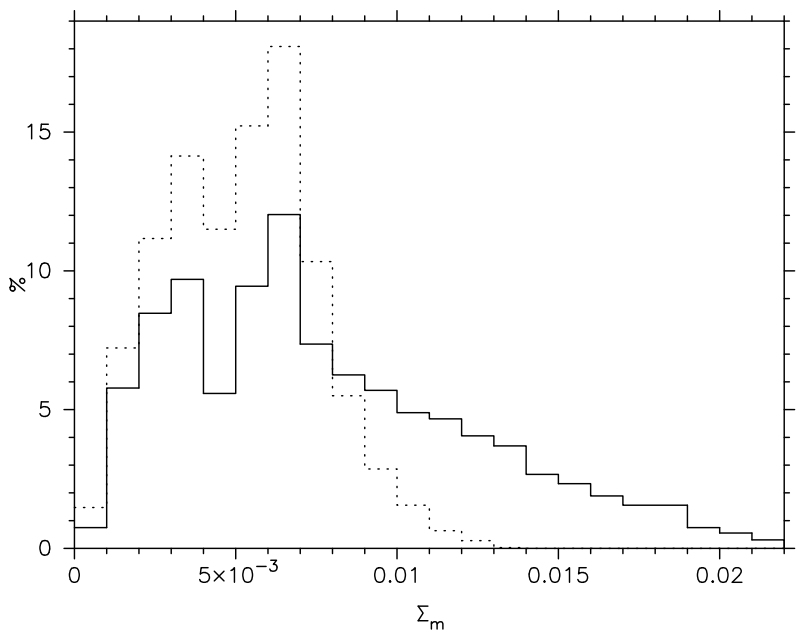

Fig. 5. Distribution of $\Sigma_{\mathrm{m}}$, which is a measure of the fit quality averaged over the line profile and expressed in continuum units - see Eq. (1), for 3600 fits without macroturbulence (full line) and with macroturbulence (either $A_{\mathrm{ISO}}, A_{\mathrm{R}}$, or $A_{\mathrm{T}}$ whichever gave the lowest $\Sigma_{\mathrm{m}}$, dotted line).

suitable to be interpreted in terms of pulsational parameters while moments are (e.g., Aerts et al. 1992; Dall et al. 2006;
Hekker et al. 2006). In practice one can use the property that the odd moments of a symmetrical line profile are zero. Thus, the values of $\langle v\rangle$ and $\left\langle v^{3}\right\rangle$ of metal lines measured with a high resolution and high $S / N$ ratio are well suited to decide if an observed line profile is subject to time-dependent line asymmetry whenever this is not obvious from visual inspection. As a guide, we provide the ranges of the values of the moments of the generated profile sets in Table 1 . The values of $\langle v\rangle$ for the six profiles shown as full lines in Fig. 3 are a: -1.1 , b: 0.8, c: -0.3 , d: -1.0 , e: -1.4 , and $\mathrm{f}: 1.9 \mathrm{~km} \mathrm{~s}^{-1}$. The corresponding values of $\left\langle v^{3}\right\rangle$ are a: -1805 , b: 226, c: 9964, d: -45658 , e: -36972 , and f: $12470 \mathrm{~km}^{3} \mathrm{~s}^{-3}$. All these values would be zero in the case of symmetrical profiles subject to white noise. The deviation is small, of the order of a few $\mathrm{km} \mathrm{s}^{-1}$, for $\langle v\rangle$, because this quantity measures the centroid of the line and thus is independent of $v \sin i$ and the microturbulence, while these two quantities do affect $\left\langle v^{3}\right\rangle$ (see Aerts et al. 1992).

The use of the odd moments for asymmetry detection has to be obtained from a few spectra spread over at least a few days in time, because line blending of course also causes a deviation from symmetry. Such deviation is time-independent, though, while the signature of pulsational broadening is always time-dependent and has typical periodicities of several hours to a few days in hot massive stars. 


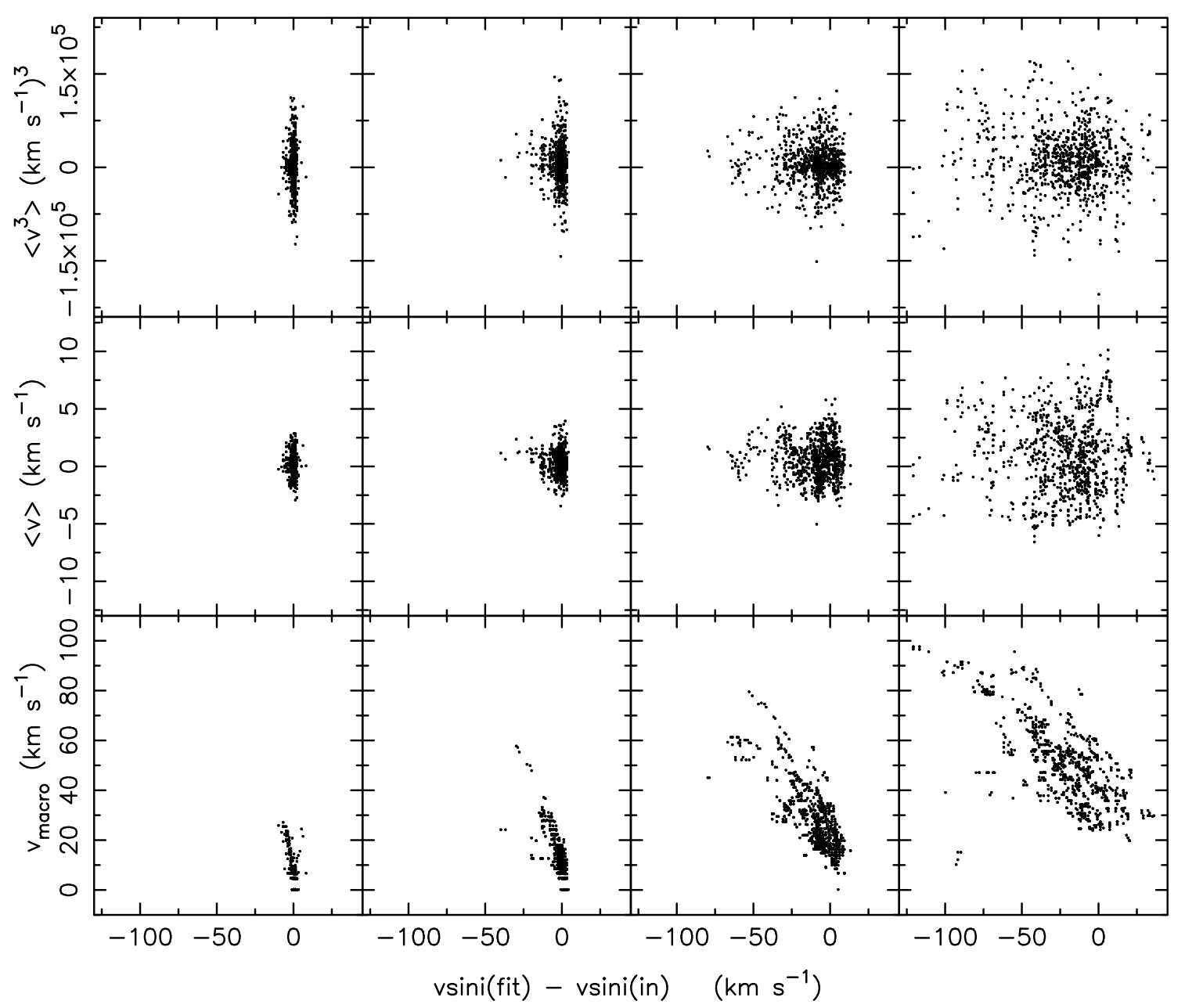

Fig. 6. The macroturbulence (either $A_{\mathrm{ISO}}, A_{\mathrm{R}}$, or $A_{\mathrm{T}}$ ) resulting in the minimal $\Sigma_{\mathrm{m}}$, as well as the first and third moments, as a function of the excess of the rotational velocity estimate compared with the input value, derived from line-profile fits ignoring the presence of pulsational broadening, for the four amplitude distributions (left to right: $a=0.1,0.2,0.5,1.0 \mathrm{~km} \mathrm{~s}^{-1}$, see also Table 1). For profiles unaffected by pulsational broadening, each of the macroturbulence, $\langle v\rangle$, and $\left\langle v^{3}\right\rangle$ values are zero.

\subsection{Consequences for the rotational velocity estimate}

An important conclusion based on Fig. 6 is that the inclusion of macroturbulence to obtain a line fit may result in a serious underestimation of the true projected rotational velocity, irrespective of which description for $v_{\text {macro }}$ is used. We encountered mismatches compared with the input $v \sin i$ above $100 \mathrm{~km} \mathrm{~s}^{-1}$. The question thus arrises if it is not wiser to exclude a $v_{\text {macro }}$ parameter to search for the best value of $v \sin i$ or to resort to other methods to determine this parameter.

The rotation velocities derived from a fit to the pulsationally broadened profiles with the inclusion of macroturbulence (we selected the version of $A_{\mathrm{ISO}}, A_{\mathrm{R}}$, and $A_{\mathrm{T}}$ which led to the lowest $\Sigma_{\mathrm{m}}$ for the plot) and without it are compared with the input $v \sin i$ in the left panel of Fig. 7. It can be seen that the mismatch of the rotation velocity ranges from -20 to $40 \mathrm{~km} \mathrm{~s}^{-1}$ for fits without allowing a parameter for macroturbulent broadening while it ranges from -120 to $40 \mathrm{~km} \mathrm{~s}^{-1}$ if a parameter for macroturbulence is allowed. From this we conclude it is better to avoid the inclusion of macroturbulence in a goodness-of-fit approach as in Eq. (1) when the goal is to achieve a good estimate of $v \sin i$. Even in that case, $v \sin i$ may be quite wrong when derived from profiles that are pulsationally broadened.

In view of the importance of having an appropriate $v \sin i$ estimate, we also resorted to the popular Fourier transform (FT) method. This method was introduced by Gray $(1973,1975)$. It was evaluated specifically for hot massive stars by Simón-Díaz $\&$ Herrero (2007). It allows one to estimate $v \sin i$ from the first minimum of the FT of a line profile. What is often forgotten, however, is that its basic assumption is that the line profiles are symmetric, which is not the case when pulsations (or other phenomena like spots) occur (e.g., Smith \& Gray 1976). We thus investigated how robust the method is when this condition is not met. We applied the method to all the 3600 pulsationally broadened profiles and derived $v \sin i$ by careful visual inspection of their FTs. A few of the FTs are shown in Fig. 9 while the global mismatch in $v \sin i$ is compared with the one obtained from the goodness-of-fit method for $v_{\text {macro }}=0$ in the right panel of Fig. 7. We see that the FT method outperformes the goodnessof-fit method when $v_{\text {macro }}$ is allowed for. On the other hand, the ability of the FT method to estimate $v \sin i$ is also affected by the pulsational broadening for a fraction of the simulated profiles and also leads to too low estimates for $v \sin i$. The offsets between the input value of $v \sin i$ and its estimate from the goodness-of-fit method with $v_{\text {macro }}=0$ on the one hand, and from the FT method on the other hand, are above $10 \mathrm{~km} \mathrm{~s}^{-1}$ in $16 \%$ and $26 \%$ of the 3600 cases, respectively. For mismatches above $20 \mathrm{~km} \mathrm{~s}^{-1}$ these numbers decrease to $6 \%$ and $12 \%$, and above $30 \mathrm{~km} \mathrm{~s}^{-1}$ a further decrease to $1.7 \%$ and $5 \%$ occurs. 

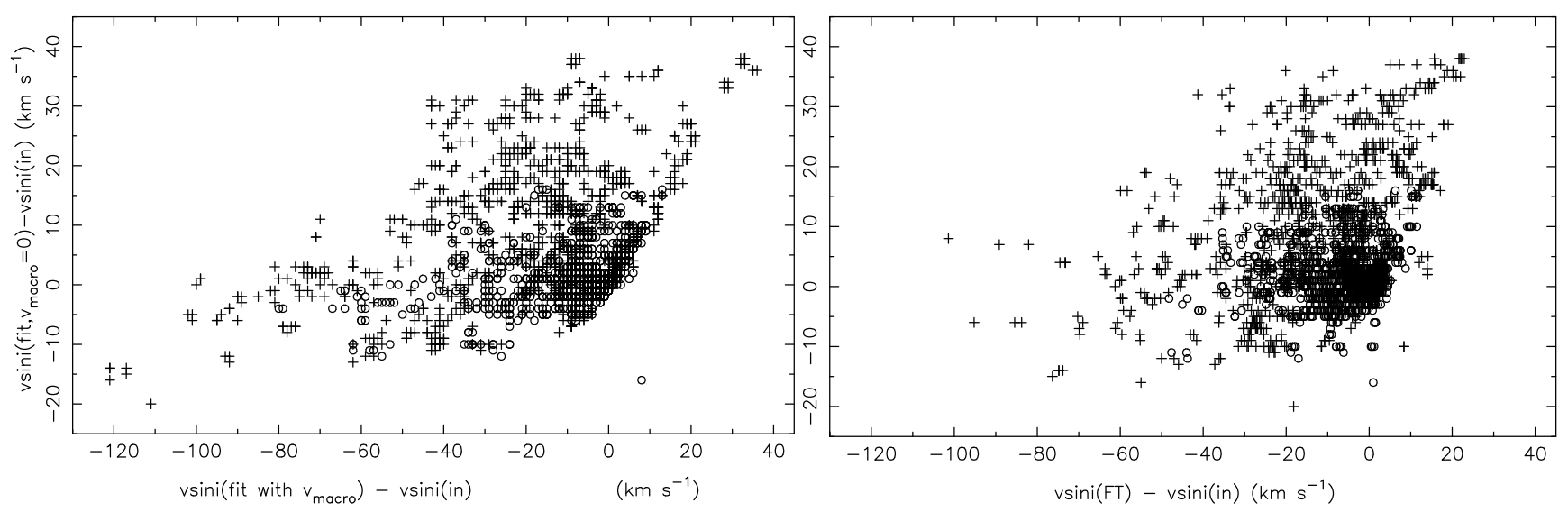

Fig. 7. Left: the estimated minus input rotation velocity from a fit without macroturbulence as a function of a fit with macroturbulence, for the simulations described in the text. The two different symbols indicate simulations for the four amplitude distributions $a=0.1,0.2,0.5(\circ)$, $1.0(+) \mathrm{km} \mathrm{s}^{-1}$ as explained in the text and listed in Table 1. Right: the estimated minus input rotation velocity from a fit without macroturbulence as a function of the value derived from the Fourier Transform method.
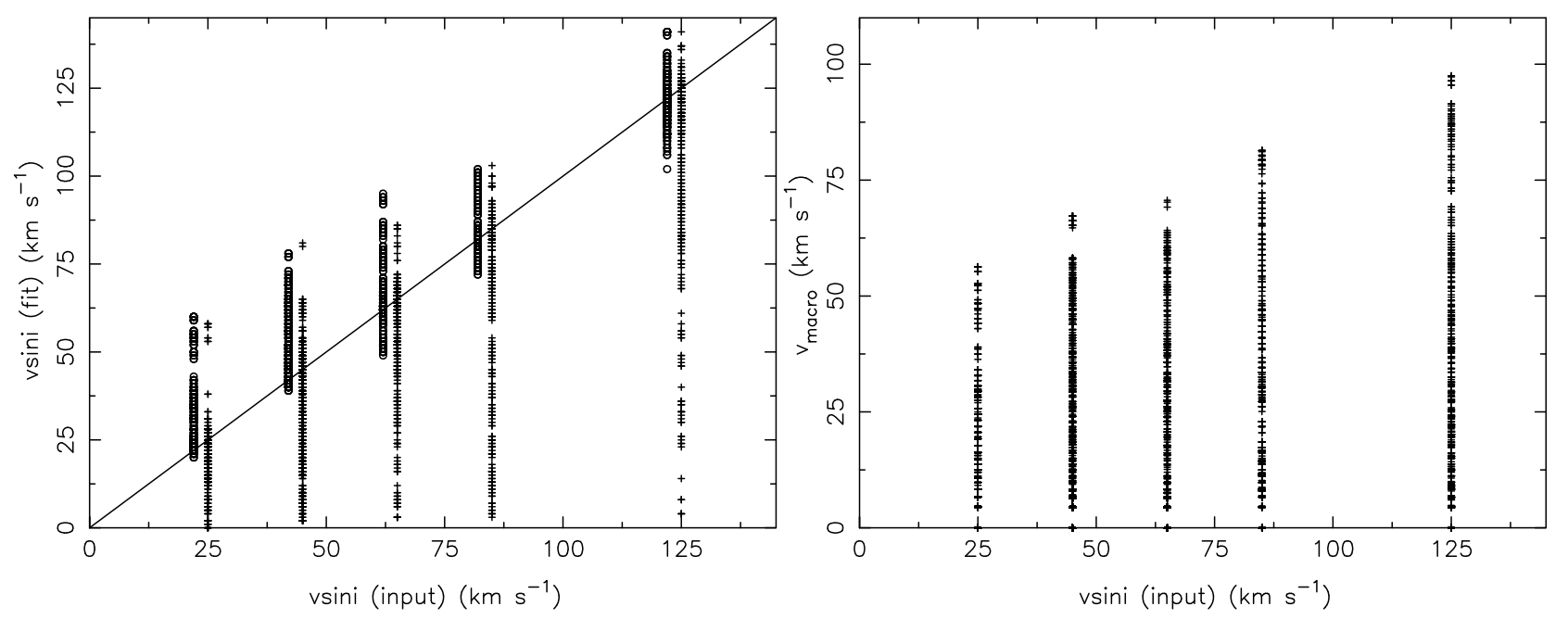

Fig. 8. Left: $v \sin i$ for fits without macroturbulence (circles) and with macroturbulence (plus signs) as a function of the input $v \sin i$, for all the simulated line profiles. The circles were artificially shifted to slightly lower input $v \sin i$ for visibility purposes. Right: the corresponding values for $v_{\text {macro }}$.

As illustrated in Fig. 9 for a few cases, the results of the FT method improve appreciably when applied to the best fit of the line profile including only microturbulent and rotational broadening, i.e., without allowing for macroturbulence. The reason is that, in this case, we approximate the true pulsationally broadened asymmetric profile by one which is symmetric and has less extended wings such that the basic assumption of the FT method is fulfilled. It was emphasized by Mihalas (1979) that the FT method has limitations of applicability when various broadening functions are convolved and result in skew profiles, which is the situation we encounter here for the gravity modes. The FT method is reliable in filtering out the value of $v \sin i$ from the observed spectral lines, when the rotational broadening is very dominant, while the pulsational amplitudes are very low (as in panel c of Fig. 9) or when broadening due to spots or pressure modes occurs, which leave the line wings almost unaltered and affect mainly the central parts of the lines.

In Fig. 8 we compare the input value of $v \sin i$ with the value deduced from a fit with and without allowing a parameter for macroturbulence; we also show the corresponding values of $v_{\text {macro }}$ for each of the simulated profiles. We find an overestimation of the rotation from $\Sigma_{\mathrm{m}}$ for low input $v \sin i$, because we need to compensate for the pulsational broadening and this can only be achieved by fitting a profile with a higher $v \sin i$ than the input value. When allowing for macroturbulence, however, we cover the entire range of projected rotation velocities between zero and values up to some $20 \mathrm{~km} \mathrm{~s}^{-1}$ above the input value of $v \sin i$, i.e., for several cases a serious underestimation of the true rotational velocity occurs. This mismatch increases with the input $v \sin i$ and occurs whenever the wings of the profiles are severaly broadened due to a positive interference of the modes with the largest horizontal velocity amplitude at some timings in the beat cycle and/or when large line asymmetries occur (see, e.g., panels d, e, f of Fig. 3 which typically have large values of $\left|\left\langle v^{3}\right\rangle\right|$ ). The right panel of Fig. 8 shows that large values of $v_{\text {macro }}$ occur for all input values of $v \sin i$, but the most extreme values for $v_{\text {macro }}$ occur typically for the broader profiles due to rotation and the larger pulsational amplitudes (see also Fig. 4).

We come to the important conclusion that, in the case of line profile broadening due to gravity modes, $v \sin i$ estimates are best derived from a simple goodness-of-fit to observed profiles, including only microturbulence and rotational broadening and no macroturbulence. 

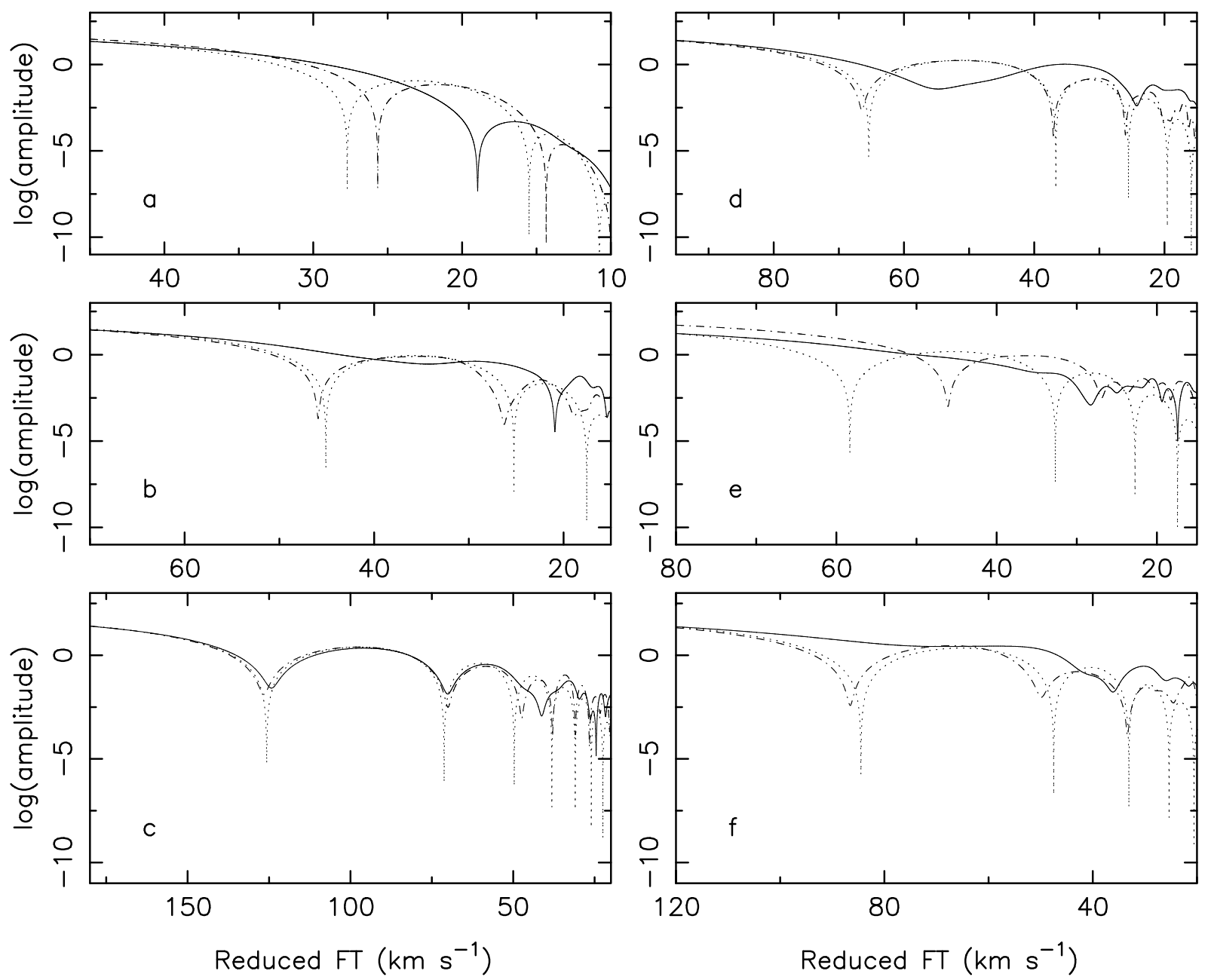

Fig. 9. Fourier transforms reduced to velocity units for the profiles in Fig. 3. The full and dotted lines have the same meaning as in Fig. 3. The dashed-dotted lines represent the results of a profile with the input rotational velocity and the same $S / N$ ratio as the full lines. By using the first (i.e., leftmost) minimum of the Fourier transform to derive $v \sin i$, profiles affected by pulsational broadening would mostly result in too low values compared to the actual (input) ones (corresponding to the first minima of the dashed-dotted lines). On the other hand, $v \sin i$ values derived from profile fits assuming $v_{\text {macro }}=0$ (dotted) often compare quite well to the actual values.

\section{Implications}

The idea that macroturbulence originates from stellar pulsations is not new. Lucy (1976) already suggested pulsations as a possibility to explain macroturbulence. Unfortunately, he did not have the observational capabilities nor the theoretical development to study the effects of pulsations on line profiles. Recent observations of massive stars with the CoRoT space mission reveal the occurrence of hundreds of pulsation modes with white-light amplitudes in the range of 0.01 and $0.1 \mathrm{mmag}$ which went unnoticed in ground-based data (Degroote et al. 2009a,b). Moreover, the discovery of massive pulsators in low-metallicity environments (e.g., Kołaczkowski et al. 2006; Narwid et al. 2006; Sarro et al. 2009) also shows that current excitation computations (Miglio et al. 2007) still underestimate the number of excited modes. Our results are thus also relevant for evolved stars in the Magellanic Clouds.

As an important side result of our study, we conclude that the rotational velocities of evolved massive stars can be seriously underestimated by using line profile fits based on a description in terms of macroturbulence. Ironically, this finding is opposite to previous arguments that, by neglecting macroturbulence, the derived $v \sin i$ values are significantly overestimated. In order to avoid erroneous estimates of $v \sin i$, we advise computing the moments of the line profiles as well as comparing the values of $v \sin i$ from fits with and without allowing macroturbulent broadening both by a goodness-of-fit approach and by the Fourier method. In this way, the probability of a wrong $v \sin i$ estimate is relatively low.

It is remarkable that the link between pulsational broadening and macroturbulence, and its effect on the derivation of $v \sin i$, was never thoroughly investigated, particularly since the surface rotational velocity derived from line profile fitting constitutes a crucial stellar parameter that is used to evaluate stellar evolution theory. Several authors, among which Hunter et al. (2008), claim to have found too low observed rotational velocities for evolved massive stars compared with theoretical predictions. Our physical model of collective pulsational broadening may help resolve this discrepancy. Accurate derivations of the rotational velocity of massive stars are also relevant in the context of Gamma-RayBurst progenitor studies (e.g., Yoon et al. 2006). In view of our results, we strongly advise the use of multi-epoch observations, because that is the best way to estimate the effect of pulsational broadening. One should attempt to take at least ten spectra with a resolution above 30000 and a $S / N$ ratio above 200, spread over different nights, and consider the broadening of different metal lines, to achieve a valid estimate of the surface rotation. 
Our present study was based on simulations of line profiles for which we considered pulsational line broadening due to velocity perturbations, while ignoring the Coriolis force. These profiles were then interpreted as due to macroturbulence. The resulting simulated profiles were constructed in such a way as to lead to realistic radial-velocity variations. It might be worth investigating how the inclusion of the collective effect of nonadiabatic temperature and gravity variations in the line-forming region of a star subject to the Coriolis force will affect the line wing broadening and its interpretation in terms of macroturbulence. Irrespective of the limitations of present line profile theory, our conclusion is clear: ignoring time-dependent pulsational line broadening in line profile fits of snapshot spectra may lead to the need to introduce an ad-hoc velocity field to account for the missing broadening in the line wings. This implies the risk of a wrong estimation of the projected rotational velocity of the star.

Acknowledgements. The research leading to these results has received funding from the European Research Council under the European Community's Seventh Framework Programme (FP7/2007-2013)/ERC grant agreement No. 227224 (PROSPERITY), as well as from the Research Council of K.U. Leuven grant agreement GOA/2008/04. The computations for this research have been done on the VIC HPC supercomputer of the K.U. Leuven. C.A. is much indebted to Dr. Leen Decin for explaining how to use the VIC and to Dr. Karolien Lefever for valuable discussions. We acknowledge suggestions from the referee which improved our paper.

\section{References}

Aerts, C. 1996, A\&A, 314, 115

Aerts, C., \& De Cat, P. 2003, Space Sci. Rev., 105, 453

Aerts, C., De Pauw, M., \& Waelkens, C. 1992, A\&A, 266, 294

Aerts, C., Mathias, P., Van Hoolst, T., et al. 1995, A\&A, 301, 781

Aerts, C., Thoul, A., Daszyńska, J., et al. 2003, Science, 300, 1926

Aerts, C., Lamers, H. J. G. L. M., \& Molenberghs, G. 2004, A\&A, 418, 639

Aerts, C., Christensen-Dalsgaard, J., \& Kurtz, D. W. 2009, Asteroseismology (Springer), in press

Briquet, M., Uytterhoeven, K., Morel, T., et al. 2009, A\&A, 506, 269
Cox, A. N., Morgan, S. M., F. J., Rogers, F. J., et al. 1992, ApJ, 393, 272 Cunha, M. S., Aerts, C., Christensen-Dalsgaard, J., et al. 2007, A\&ARv, 14, 217 Dall, T. H., Santos, N. C., Arentoft, T., et al. 2006, A\&A, 454, 341

Degroote, P., Aerts, C., Ollivier, M., et al. 2009a, A\&A, 506, 471

Degroote, P., Briquet, M., Catala, C., et al. 2009b, A\&A, 506, 111

De Ridder, J., Dupret, M.-A., Neuforge, C., et al. 2002, A\&A, 385, 572

Dupret, M.-A. 2001, A\&A, 366, 166

Dupret, M.-A., De Ridder, J., Neuforge, C., et al. 2002, A\&A, 385, 563

Gough, D. O., Kosovichev, A. G., Toomre, J., et al. 1996, Science, 272, 1296

Gray, D. F. 1973, ApJ, 184, 461

Gray, D. F. 1975, ApJ, 202, 148

Gray, D. F. 1978, Sol. Phys., 59, 193

Gray, D. F. 2005, The Observation and Analysis of Stellar Photospheres, 3rd edn (Cambridge University Press)

Hekker, S., Aerts, C., De Ridder, J., et al. 2006, A\&A, 458, 931

Howarth, I. D., Siebert, K. W., Hussain, G. A. J., et al. 1997, MNRAS, 284, 265

Hunter, I., Lennon, D. J., Dufton, P. L., et al. 2008, A\&A, 479, 541

Kaufer, A., Stahl, O., Wolf, B., et al. 1997, A\&A, 320, 273

Kołaczkowski, Z., Pigulski, A., Soszyński, I., et al. 2006, Mem. Soc. Astron. Ital., 77, 336

Lee, U. 2001, ApJ, 557, 311

Lefever, K., Puls, J., \& Aerts, C. 2007, A\&A, 463, 1093

Lucy, L. B. 1976, ApJ, 206, 499

Markova, N., \& Puls, J. 2008, A\&A, 478, 823

Markova, N., Prinja, R. K., Markov, H., et al. 2008, A\&A, 487, 211

McErlean, N. D., Lennon, D. J., \& Dufton, P. L. 1998, A\&A, 329, 613

Miglio, A., Montalbán, J., \& Dupret, M.-A. 2007, MNRAS, L21

Mihalas, D. 1979, MNRAS, 189, 671

Morel, T., Butler, K., Aerts, C., et al. 2006, A\&A, 457, 651

Narwid, A., Kołaczkowski, Z., Pigulski, A., et al. 2006, MmSAI, 77, 342

Pamyatnykh, A. A. 1999, Acta Astron., 49, 119

Prinja, R. K., Rivinius, Th., Stahl, O., et al. 2004, A\&A, 418, 727

Ryans, R. S., Dufton, P. L., Rolleston, W. R. J., et al. 2002, MNRAS, 336, 577

Saesen, S., Briquet, M., \& Aerts, C. 2006, CoAst, 147, 109

Saio, H., Kuschnig, R., Gautschy, A., et al. 2006, ApJ, 650, 1111

Sarro, L. M., Debosscher, J., Lopez, M., et al. 2009, A\&A, 494, 739

Scuflaire, R., Théado, S., Montalbán, J., et al. 2008, Ap\&SS, 316, 149

Simón-Díaz, S., \& Herrero, A. 2007, A\&A, 468, 1063

Smith, M. A., \& Gray, D. F. 1976, PASP, 88, 809

Štefl, S., Aerts, C., \& Balona, L. A. 1999, MNRAS, 305, 505

Townsend, R. H. D. 2005, MNRAS, 360, 465

Villamariz, M. R., \& Herrero, A. 2000, A\&A, 357, 597

Yoon, S.-C., Langer, N., \& Norman, C. 2006, A\&A, 460, 199 